\title{
COVID-19 INFECTION IN INFLAMMATORY BOWEL DISEASE PATIENTS TREATED WITH TNF-A ANTAGONISTS: A POSSIBLE CRITICTAL ENROLLMENT OF GUT MICROBIOTA AND VITAMIN D LEVEL - A REVIEW
}

\author{
ALSHEHRI, D. $.^{1,2^{*}}-$ MOSLI, M. ${ }^{3,4}-$ BAHIELDIN, A. ${ }^{1,5}$ \\ ${ }^{I}$ Department of Biological Sciences, Faculty of Science, King Abdulaziz University, Jeddah \\ 21589, Saudi Arabia \\ (phone/fax: +966-2-460-0000) \\ ${ }^{2}$ Department of Biological Sciences, Science Collage, University of Tabuk, Tabuk, Saudi Arabia \\ ${ }^{3}$ Department of Medicine, King Abdulaziz University, Jeddah, Saudi Arabia \\ (phone: +966-12-640-8272; fax: +966-12-695-2538) \\ ${ }^{4}$ Inflammatory Bowel Disease Research Group, King Abduklaziz University, Jeddah, Saudi \\ Arabia \\ (phone: +966-12-640-8272; fax: +966-12-695-2538) \\ ${ }^{5}$ Department of Genetics, Faculty of Agriculture, Ain Shams University, Cairo, Egypt \\ *Corresponding author \\ e-mail: dalshehri@ut.edu.sa; phone: +966-55-562-0021; fax: +966-2-460-0000
}

(Received $1^{\text {st }}$ Aug 2020; accepted 22 $2^{\text {nd }}$ Oct 2020)

\begin{abstract}
The ongoing pandemic of coronavirus disease 2019 (COVID-19) is emerging as a public health crisis worldwide. Patients with COVID-19 range from being asymptomatic to suffering from severe pneumonia, acute respiratory distress syndrome (ARDS), and multiple organ failure (MOF). Gastrointestinal (GI) symptoms have been reported in a number of patients with COVID-19, suggesting that GI microbiota may play a role in the pathogeneses of the disease. Theoretically, patients with confirmed inflammatory bowel disease (IBD) treated with immune-based therapies may be at a higher risk of manifesting a severe form of COVID-19, owing to immune system impairment. This hypothesis has evoked the concerns of patients and treating physicians throughout the pandemic. However, surprisingly, the findings of a number of studies show that immunosuppressive therapies, such as antiTNF agents, could reduce the severity of symptoms associated with Covid-19. Dysbiosis of gut microbiota, characteristic of IBD patients, can be positively changed after using anti-TNF agents. Vitamin $\mathrm{D}$ has been revealed to have a profound effect on reducing the viral infections, aside from its role in modulating the gut microbiome. In this review, we discuss possible susceptibility of IBD patients to SARS-CoV-2 infection, the impact of immunosuppressive therapies on the course of SARS-CoV-2 infection in patients with IBD, and the potential protective role of gut microbiota against COVID-19 in patients with IBD in the presence of normal vitamin D levels.
\end{abstract}

Keywords: Crohn's disease, ulcerative colitis, SARS-CoV-2, IBD, biologics, microbiome, VDR

Abbreviations: IBD: inflammatory bowel disease, COVID-19: corona virus disease that is a novel virus detected in 2019, CD: Crohn's disease, UC: ulcerative colitis, SCFAs: short chain fatty acids, VDR: vitamin D receptors, MOF: multi organ failure, ARDS: acute respiratory distress syndrome, MODS: multiple organ dysfunction syndrome, GI diseases: gastrointestinal diseases, ICU: intensive care unit, HCoV: human corona viruses, IFX: infliximab, ADA: adalimumab, Anti-TNF: anti-tumor necrosis factor, ARBs: angiotensin receptor blockers 


\section{Background}

The challenge of controlling this infectious disease stems from the rapid spread of the causative virus, severe acute respiratory syndrome coronavirus 2 (SARS-CoV-2), across all nations and across all borders. The emergence of the severe acute respiratory syndrome coronavirus 2 (SARS-CoV-2) infection was first reported in December 2019 in Wuhan, central Hubei Province, China and has subsequently spread worldwide (Wang et al., 2020). As of mid-June 2020, approximately 10 million cases and 500000 deaths in 200 countries have been reported (https://www.worldometers.info/coronavirus/). Ten percent of patients have required hospitalization or admission to an intensive care unit (ICU) (Danese et al., 2020; Huang et al., 2020). This has resulted in a dramatic challenge for governments and services, putting significant pressure on healthcare providers owing to the potential for exceeding the capacity of healthcare systems around the world, including IBD centers

Notably, the gastrointestinal (GI) tract can be a transmission route for SARS-CoV-2, as some GI warning signs, such as nausea, diarrhea, and abdominal pain have been recorded in about 20-50\% of COVID-19 patients (Lin et al., 2020; Zang et al., 2020). These symptoms likely appear before the development of respiratory symptoms, indicating that the GI tract may be a target organ for the virus. Therefore, patients suffering from intestinal disorders, particularly IBD, could potentially be vulnerable to COVID-19 infection in the current epidemic.

During the course of the disease, a large number of patients with IBD receive biologics treatments such as $\alpha$-TNF antagonists, which have double-edged sword effects. These medications contain molecules that can cause inflammation, increasing the risk of infections such as respiratory illnesses as a result of immune system impairment (Dipasquale and Romano, 2018). This can be complicated with dynamic bidirectional interactions with the host microbiome. Although recent studies have suggested that most immune system responses are driven by the host genome's stimulations, about $10 \%$ of immune system responses were reported to be stimulated by a direct interaction with the host microbiome (Gilbert et al., 2018; Schirmer et al., 2016). The GI microbiota is documented to have an effect on the quality of the host immune system, which participates, in turn, in modulating gut microbiota composition. Prior studies have approved the major influence of nutrient substances and diet on GI microbiota composition and localization, and their link with immunological pathways (Gao et al., 2018; Thorburn et al., 2014). For example, vitamin D was found to be linked to an individual's GI microbial diversity and to healthy interactions between GI microbiota and intestinal immunity, including short chain fatty acid (SCFA) butyrate (Celiberto et al., 2018).

Supportive evidence has led to speculation about the role of GI microbiota and vitamin D in COVID-19 patient outcomes. It has been observed that individuals at higher risk of evolving severe COVID-19 are those aged $>60$ years and with underlying chronic diseases, such as hypertension, diabetes, cardiovascular illness, chronic respiratory illness, and cancer (Alifano et al., 2020) - all of which are known to coincide with GI microbiota dysbiosis. Also, elderly people have been shown to suffer more often from severe deficiency in vitamin D (Kweder and Eidi, 2018).

In both humans and mice, it has been found that the expression of the angiotensin Iconverting enzyme 2 (ACE2), to which SARS-Cov-2 binds when entering the cell, is considerably higher in the small intestine than in other organs, including the lungs (Zang et al., 2020). Thus, it may be important to investigate the degree of susceptibility 
of IBD patients to COVID-19 infection. Other clinically relevant questions include whether the use of immune-based therapies has a negative effect on prognosis, or otherwise can help in fighting COVID-19 complications on one hand, or whether gut microbiota composition and vitamin D level have a protective role against COVID-19 infection on the other hand.

\section{SARS-CoV-2 and COVID-19 infection across the globe}

Severe acute respiratory syndrome coronavirus 2 belongs to the Coronaviridae ( $\beta$ coronavirus) family, which share the characteristics of being single-stranded, positive sense (+ ssRNA) viruses and have an enveloped glycoprotein surface that appears like a crown under microscope. This family includes the largest identified RNA viruses, which extend between 26 to $32 \mathrm{~kb}$ in length (Lai and Cavanagh, 1997; Weiss and Navas-Martin, 2005). For unknown reasons, coronaviruses, like MERS and SARS, have the ability of pass through species barriers causing diseases in humans, which range from being asymptomatic to more severe signs and symptoms. These viruses, termed human coronaviruses (HCoVs), were first discovered in 1960, and shown to be transferred to mammals from various other organisms (Al-Ani et al., 2020). Other novel viruses of the Coronaviridae family may arise in the future, because these viruses interact together in nature. This makes genetic recombination among their genetic materials simple and therefore likely (Su et al., 2016). As the virus rapidly evolves, we need to investigate whether or not it continues to be as virulent.

To date, there are seven identified HCoVs. The latest $\mathrm{HCoV}$ is proposed to be originated from bats and transferred to other mammals before affecting humans (Cascella et al., 2020). Examples of mammals that have been infected by HCoVs that have crossed the species barrier are the Himalayan palm civet, which was found to have a form of SARS-CoV ( $79 \%$ genomic similarity to SARS-Cov-2), and the dromedary camel, which was infected with MERS-CoV ( $50 \%$ genomic similarity to SARS-Cov2) (Cao and Li, 2020). However, the dynamics of SARS-Cov-2 are still unclear; it has been speculated that the virus might be transmitted to humans from other animals (Fig. 1) (Su et al., 2016). Routine viral transmission between hosts has not been well documented for HCoVs, however, it seems that they are much more transmissible among humans (Zhou et al., 2020). Among human individuals, transmission can occur either by direct contact or through the air, or possibly indirectly through touching inanimate surfaces contaminated with the virus. It is unclear how long the virus can survive on or in non-biological materials, but approximately nine days is estimated to be the survival time for most of these viruses; by contrast, influenza virus can survive on surfaces for many months (Conti et al., 2020).

On 28 February, 2020, the World Health Organization (WHO) raised the threat level of the SARS-Cov-2 epidemic to "very high", followed by an announcement on 11 March that the COVID-19 pandemic is a very high risk to health owing to its rapid spread internationally (Cascella et al., 2020). The WHO's chief executive, Tedros Ghebreyesus, has criticized nations that made no adequate tests for their populations, or had limited testing (Al-Muharraqi, 2020). A number of countries, owing to capacity problems regarding COVID-19 test availability, initially restricted testing to individuals that suffer from severe acute respiratory syndromes (Al-Muharraqi, 2020). The problem of asymptomatic patients, the high infectivity of SARS-Cov-2, a lack of an effective 
vaccine or antiviral, and late quarantine decisions in many countries contributed to the sharp increase in the number of affected individuals worldwide.

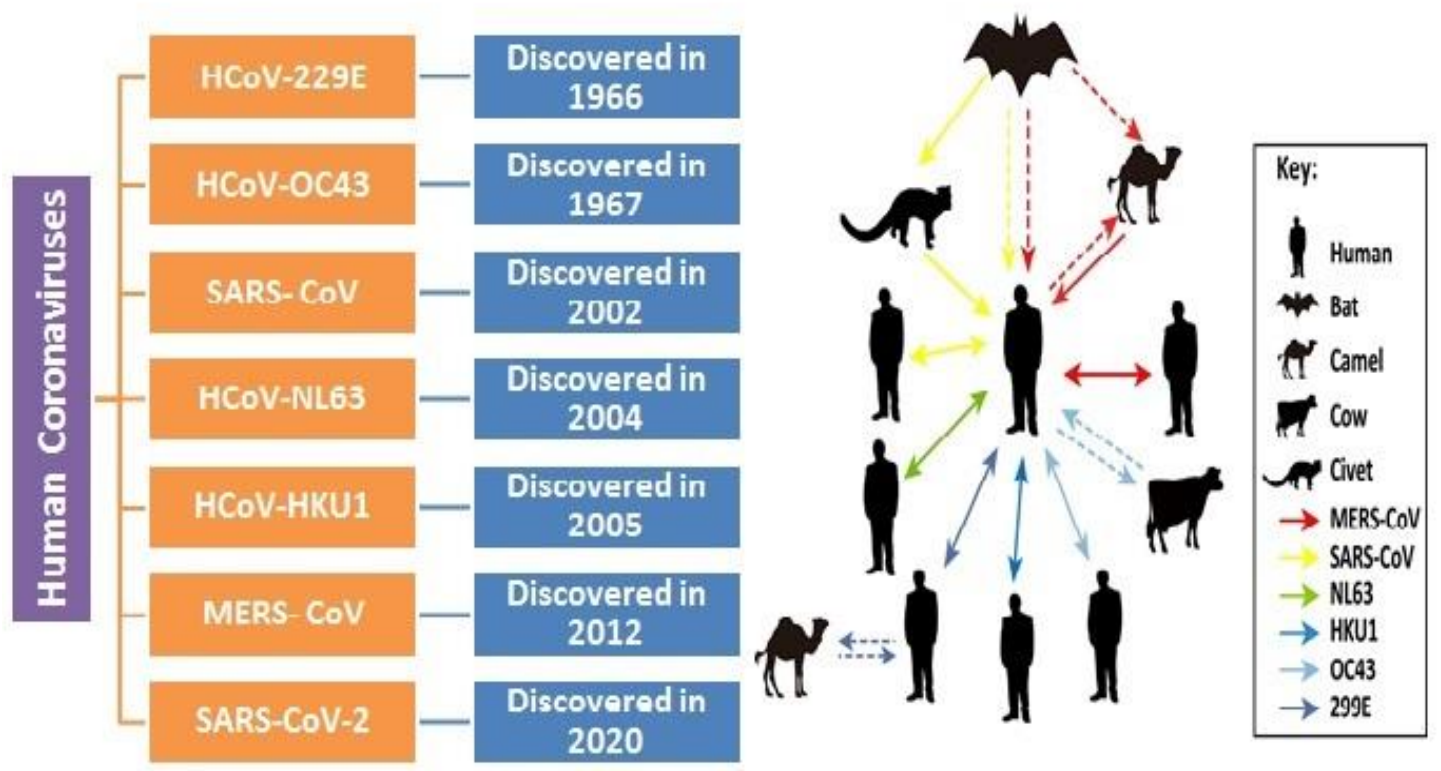

Figure 1. The seven reported human coronavirus (HCoVs) and intra- and inter-species transmission of human coronaviruses. Figure was taken after Su et al., 2016

Recent reports have indicated that elderly individuals, in particular those treated with immune-suppressives or biologic therapies or have a history of coronary artery disease, are more prone to a severe form of the disease and a higher mortality rate compared to other age groups, such as children and adolescents (Monteleone and Ardizzone, 2020). These findings have shown an almost null death rate in children between 0 and 10 years, and $0.2 \%$ death rate in children between 10 and 19 years (Huang et al., 2020; Zhu et al., 2020). Also, SARS-Cov-2 is believed to affect males more than females, possibly owing to the large number of immune genes located on the X chromosome (Conti et al., 2020; Ghosh and Klein, 2017).

\section{Invasion of the host cells by SARS-CoV-2 and COVID-19 symptoms}

Similar to SARS-CoV-1, SARS-CoV-2 can enter human cells via the reninangiotensin system (Guo et al., 2020; Rico-Mesa et al., 2020). This system comprises of two main enzymes that control peptide balance in the angiotensin family. These two enzymes are angiotensin I-converting enzyme 2 (ACE2), expressed in the respiratory epithelium, intestines, and macrophages (Lu et al., 2020), and transmembrane serine protease 2 (TMPRSs2). Both enzymes are important for viral infectivity (Hoffmann et al., 2020). The successful isolation of a sub-genomic messenger RNA (sgRNA) of the live virus from throat swabs indicates that SARS-CoV-2 has the ability to actively replicate in upper respiratory tissue cells (Lu et al., 2020; Wölfel et al., 2020). However, similar to SARS-CoV-1, CoV-SARS 2 firstly replicates in the lower respiratory tissues. Following this, organs with high expression of ACE2 (such as heart, kidney, 
gastrointestinal tract, and vast distal vasculature) start to be aggressively attacked by the virus, specifically in the later stage of the disease course-generally the second week following the onset of COVID-19 (Cao and Li, 2020). Other significant complications induced by SARS-CoV-2 have been recognized, such as direct viral damage and consequences of immune-mediated injury. Of particular note, sever acute cases of COVID-19 often have two critically distinctive features, which are progressive increase of inflammation and the unusual trend of hyper-coagulation (Cao and $\mathrm{Li}, 2020$ ).

Clinically, severity of SARS-CoV-2 can range from asymptomatic to very severe pneumonia, leading to acute respiratory distress syndrome or multi-organ failure (MOF). Many patients develop dyspnea and hypoxemia, leading to mild respiratory syndromes that require $\mathrm{O}_{2}$ therapy. Others, however, rapidly develop acute respiratory distress syndromes (ARDSs), resulting on occasion in septic shock, metabolic acidosis, coagulation dysfunction with disseminated intravascular coagulation (DIC), and multiple organ dysfunction syndrome (MODS) (Singhal, 2020). However, it is broadly acknowledged that at present there is no indication about which individuals are highly susceptible from being seriously negatively affected from COVID-19. Broadly, SARSCoV-2 could affect any individual, causing acute respiratory symptoms that can result in death. Nearly one in four infected individuals require intensive healthcare and approximately $4.3 \%$ of infected patients eventually die (Wang et al., 2020). Thus, COVID-19 is a serious and complex disease that is multi-faceted, involving all the systems and organs of the human body.

Regarding IBD patients, the expression of ACE2 was reported to increase in inflamed intestines (Garg et al., 2020). Proteomic investigations showed higher expression of ACE2 in Crohn's disease (CD) compared to Ulcerative colitis (UC) (Ning et al., 2019). The invasion of SARS-CoV-2 in a cell is mediated by a specific type of fusion, using a 'spike' protein (Ibrahim et al., 2020). This protein is activated via a proteolytic cleavage, triggered by the host cell using trypsin-like proteases: enzymes that are demonstrated to be upregulated in IBD patients (Jablaoui et al., 2020). This evidence suggests that the increased probability of inflamed intestinal tissues in IBD patients may be an optimal environment for SARS-CoV-2 activity. Nevertheless, no research has yet examined the frequency of COVID-19 occurrence in IBD patients.

\section{Immune-based medication in IBD patients with COVID-19: is there a risk of increased occurrence and severity?}

There is no clear evidence that patients confirmed with IBD are more susceptible to developing severe COVID-19 symptoms. Nevertheless, there are indications in previous studies that IBD patients that take immune-suppressive drugs are at high risk of having infectious diseases, possibly since the intracellular signaling cascades needed for the host to resist pathogens are inhibited (Beaugerie et al., 2020; Holmer and Singh, 2019; Singh et al., 2020). Earlier reports indicate that infections of upper and lower respiratory tract increased when using anti-TNF therapies, besides other several opportunistic pulmonary infections (Lichtenstein et al., 2012; Long et al., 2013; Shah et al., 2017). A study conducted on IBD patients to assess the increasing risk of pneumonia infection indicated that the use of biologics resulted in an increased risk (OR 1.28, 95\% CI 1.081.52), and corticosteroids showed a significant increase (OR 3.62, 95\% CI 3.30-3.98) (Singh et al., 2020). However, the study did not highlight if the medications were combined or not. 
A recent systemic review with a meta-analysis of clinical trial data, which included 4135 patients that underwent anti-TNF treatment (Ford and Peyrin-Biroulet, 2013), concluded that individuals are at risk of doubling bacterial and viral infectivity (such as tuberculosis, herpes simplex infection, oral or esophageal candidiasis, herpes zoster virus, cytomegalovirus, and Epstein-Barr virus) with anti-TNF treatment (OR 2.05, 95\% CI $1.10-3.85$ ) compared to a placebo. Consequently, the monotherapy of anti-TNF therapy seems to be safer than in combination with other immunosuppressive agents. Importantly, a significant increased risk of developing infectious disease is shown when combining anti-TNF treatments with corticosteroids (Singh et al., 2020; Ye et al., 2020). This is because the early induction of corticosteroids can eliminate the initiation of the host immune system defense, possibly leading to adverse consequences.

In contrast to the above findings, recent research has examined the role of anti-TNF therapy as a treatment for COVID-19. Many pro-inflammatory cytokines, such as interleukin (IL)-1, IL-6, TNF, and interferon $\gamma$ (Gong et al., 2020; Huang et al., 2020), have been reported to be upregulated in the blood of patients confirmed with COVID-19 (Monteleone and Ardizzone, 2020). Patients hospitalized under intensive care showed high concentrations of a number of cytokines. Furthermore, some other cytokines and chemokines are deregulated in the plasma of affected cells, causing what is generally termed a 'cytokine storm'. TNF and IL6 are well noted in acute respiratory conditions to potentially lead to lunge injury (Kovalchuk et al., 2020). In animal studies, TNF cytokines have been shown to contribute to the development of lung injury and weakening of $\mathrm{T}$ cell response in mice infected with SARS-CoV. The loss of TNF receptors and the neutralization of the activity of TNFs have resulted in protection against the morbidity and mortality associated with SARS-CoV (Channappanavar et al., 2016; McDermott et al., 2016). Accordingly, many potential therapeutic agents were nominated for controlling inflammation in COVID-19, of which, anti-TNF antagonists (infliximab IFX or adalimumab ADA, which have been approved, and widely available) are superior, because they show potential in being effective, particularly for acute inflammation cases (Feldmann et al., 2020). Clinically, in many inflammatory diseases and conditions, the blockage of TNF cytokines is effective, despite the existence of other pro-inflammatory cytokines (Feldmann et al., 2020). Therefore, the effectiveness of anti-TNF antibodies in treating COVID-19 warrants further consideration, especially when we know that TNFs are not detected in the serum of SARS-affected patients at later stages (Ye et al., 2020). Currently, $\alpha$-TNF antagonists have not been used to treat patients with COVID-19, but it requires further exploration.

\section{GI microbiota composition and vitamin D level in IBD patients with COVID-19 treated with TNF antagonists}

Since the use of biologics to treat IBD can result in impairment of the immune system (Dipasquale and Romano, 2018), which can lead to an increased possibility of infection, it is important to investigate the probability of infection of IBD patients with COVID-19 that have been treated with TNF antagonists. Reducing the gut microbiota diversity, known as dysbiosis, has been well documented in IBD patients (Sartor, 2006), increasing the number of pro-inflammatory cytokines, such as Escherichia coli. (Celiberto et al., 2018). Onset and progression of IBD has been shown to be associated with insufficient serum vitamin D levels (30-75 nmol/L). Almost $68 \%$ of IBD patients suffer from vitamin $\mathrm{D}$ insufficiency, and more than $50 \%$ of those suffer from vitamin D deficiency $(<30$ 
nmol/L) (Celiberto et al., 2018; White, 2018). Vitamin D has been shown to be of considerable importance in immune modulation, with a potential to improve IBD and COVID-19 patients (Celiberto et al., 2018; Grant et al., 2020). It can modulate proinflammatory Th1 and Th17 cells, which express TNF $\alpha$, interferon- $\gamma$ and IL-17, similar to those that appear in COVID-19 cytokines storms (Wu and Yang, 2020).

CoV-SARS-2 was documented to use ACE-2 receptors for cellular entry (Zhou et al., 2020), and, as noted above, the receptors can be highly expressed in the small intestine over other organs, including the lungs (Leung et al., 2003; Zang et al., 2020). In addition to this, the feces-oral route has also been suggested (Zang et al., 2020), with the higher mortality rate of the elderly affected with COVID-19 (Liu et al., 2020), and those elderly individuals previously documented to have less diverse GI microbiota composition (Claesson et al., 2011, 2012; Yatsunenko et al., 2012) and often suffer from deficient vitamin D levels (Kweder and Eidi, 2018). Recent literature showed that COVID-19 has an association with increased pro-inflammatory cytokines (Wang et al., 2020), whereas the immune-modulatory properties owing to vitamin D can downregulate the pro-inflammatory cytokines (Arboleda et al., 2019; Greiller and Martineau, 2015; Panarese et al., 2019; Zdrenghea et al., 2017; Zhang et al., 2012). Furthermore, vitamin D has shown to have the ability of preventing lung injury via blocking these observations, with the evidence that vitamin D-VDR signaling prevents lung injury by blocking effects on the renin-angiotensin pathway (Kong et al., 2013).

In a cohort study conducted in the US, approximately $61 \%$ of COVID-19 patients manifest GI symptoms (Redd et al., 2020). Also, the RNA of CoV-SARS-2 was shown to be detected in patient stools (Cheung et al., 2020; Holshue et al., 2020; Wu et al., 2020; Xiao et al., 2020; Zhang et al., 2020). Another study found that vitamin D signaling has a critical role in healthy association between the GI tract microbiota and intestinal immunity, including the SCFAs in particularly butyrate, which increases the vitamin D receptors (VDRs) in vitro. Also, butyrate can directly bind to the VDR, resulting in down-regulation of TNF- $\alpha$ production (Schwab et al., 2007). Altogether, this data led suggests a critical role for gut microbiota and vitamin $\mathrm{D}$ in fighting against COVID-19 infections and limiting the severity of the disease.

There are complex interaction networks among GI microbiota composition and the host immune system, which are needed for a physiological microbiota-host balance, which have a profound effect on health and disease (Lagkouvardos et al., 2017). Dysbiosis in the gut microbiota composition has been linked to many disorders; one of these is IBD (De Musis et al., 2020; Khan et al., 2019; Lavelle and Sokol, 2020). Pathogen-induced microbiota can diminish the chance of beneficial bacteria colonization, leading to inflammation development. Similar to GI microbiota, research has shown that the lungs have distinct microorganisms (Bingula et al., 2017; Zhang et al., 2020). Interestingly, pulmonary health has been shown to be affected by gut microbiota composition, through a vital 'cross-talk' between the gut and lungs, known as the 'gut-lung axis' (Keely et al., 2012). The latter is thought to be a bidirectional relationship, as the effects of endotoxins and metabolites of gut microbiota, on one hand, and lung inflammation, on the other hand, is mutual (Dumas et al., 2018). Several studies have illustrated the association between respiratory infections with GI microbiota alterations (Groves et al., 2020). Interestingly, this attests to the effect of SARS-Cov2 infection on gut microbiota composition and the converse, since patients confirmed with COVID-19 have been found to suffer from ARDS, particularly elderly and immune-compromised individuals (Lake, 2020). 
The relationship between lung infections including COVID-19 and potential role of healthy gut microbiome were speculated in recent studies that have explored the facesoral route. A study conducted in Hong Kong covered 59 patients with confirmed COVID-19 $(\mathrm{n}=59)$, where 15 individuals $(25.4 \%)$ had GI symptoms and 9 individuals (15.3\%) had tested positive for SARS-CoV-2 in their stool RNA. The results are more pronounced if the patient was suffering from diarrhea $(38.5 \%$ and $8.7 \%$ in patients with and without diarrhea, respectively) $(P=0.02)$ (Cheung et al., 2020). They searched for supplementary meta-analysis data published in various databases concerning patients with GI symptoms and the presence of SARS-CoV-2 RNA in their stools. Approximately $17.6 \%$ of patients were shown to have GI symptoms, and SARS-CoV-2 was detected in the genetic material of stool samples of about $48.1 \%$ of patients, after being found negative in their previous respiratory tests (Cheung et al., 2020). Another study conducted in February 2020 targeted 73 hospitalized patients confirmed with COVID-19 aged between 10 months and 78 years old to test the GI infection and presence of SARS-CoV-2. The analysis of the patients stool RNA showed that 39 (54.42\%) of tested samples were positive for SARS-CoV-2. The positivity of the test lasts from 1-12 days. However, 17 patients (23.29\%) that had tested negative for respiratory infection continued to show positive results in their stools (Xiao et al., 2020). This enhances the possibility of fecal-oral transmission, where the virus targets GI tract, corroborating the findings that viral RNA causing COVID-19 has been detected in feces of patients, despite testing negative for respiratory infection (Effenberger et al., 2020; Zang et al., 2020). The detected virus was described to be non-infective (Zang et al., 2020), which drives our speculation that this impaired virus may have potential for use in the production of an effective vaccine against the infecting version of the virus.

The use of biologics, such as anti-TNF treatments in IBD patients with less diverse gut microbiota alterations, was shown to improve the microbiota profile in responders (Alshehri et al., 2020). This was found in a study carried out on Chinese pediatric patients with Crohn's disease (CD), which were treated using IFX. The microbial dynamic changes were assessed during treatment and investigated for the influence of the anti-TNF agent on the microbiota composition. The use of IFX was shown to alter gut microbiome structure and metabolic function, diminishing CD-correlated GI microbial dysbiosis, accompanied with a significant increase in the number of beneficial bacteria (Li et al., 2017). This may indicate that using an anti-TNF agent can be of great help for improving the GI microbiota profile, as well as a defense against COVID-19, which come along with the importance of and normalizing the vitamin D level.

\section{Further recommendations}

1. It seems that there is no documented evidence on the predisposition of IBD patients that undergo biologics treatment, such as anti-TNF agents, to COVID19. Conversely, anti-TNF agents may be an effective therapy against SARSCoV-2.

2. Physicians should update (at least temporarily) their viral screening lists for IBD patients to include testing for SARS-CoV-2, besides the other tests, such as hepatitis B, hepatitis C, HIV, varicella zoster, and tuberculosis, before starting biologics or other immune-based therapies. This is because of the confirmation on the susceptibility of IBD patients to infectious illnesses and 
the detection of the SARS-CoV2 virus in stool samples of COVID-19 patients, especially those with diarrhea.

3. The intersection between COVID-19 infection, IBD course, and effective usage of immune-based therapies is yet to be fully explored. However, physicians could carefully make individual risk assessments of patients before initiating immune-suppressive drugs therapies with IBD patients to potentially avoid complications, especially if they are combining drugs. Physicians should avoid combination therapies, in particular, using anti-TNF agents with corticosteroids.

4. Improving the GI microbiota profile and vitamin D levels deserve further consideration for improving the fight against COVID-19. Furthermore, additional studies on infection caused by SARS-Cov2 are required to address the intervention of intestinal and lung commensal microorganisms (Fig. 2).

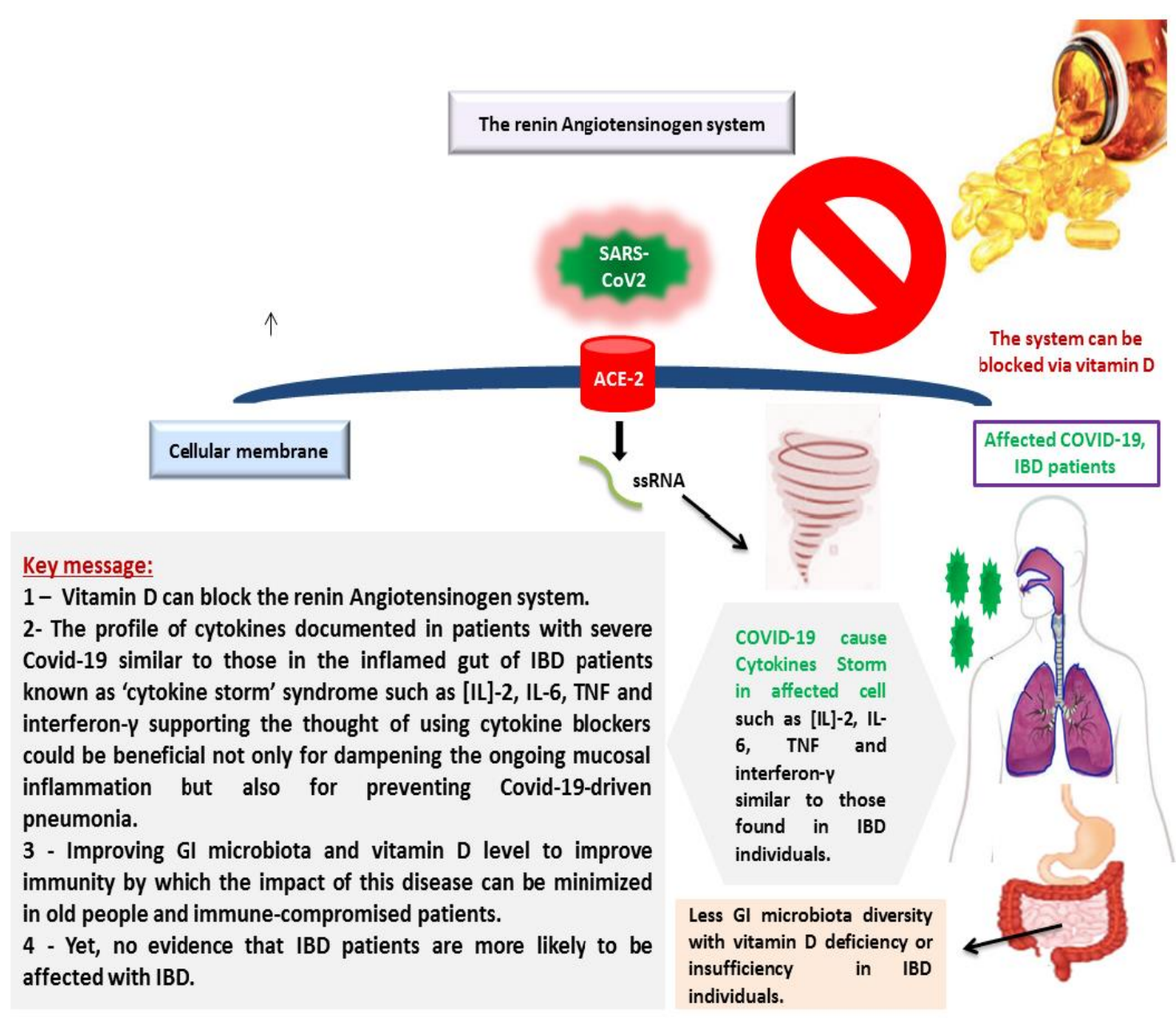

Figure 2. Summary and key messages from this review

\section{Conclusions}

It has been reported that using biologics and immunomodulators can impair the patient's immune system; immunotherapies used to treat IBD may increase susceptibility to COVID-19 infection. However, infection with COVID-19 causes a 
cytokines storm that can result in hyper-induction in various pro-inflammatory cytokines of the immune system, which may be modulated using immunomodulators such as $\alpha$-TNF antagonists and immune-nutrition, such as vitamin D. Since the gut microbiota and vitamin D are acknowledged to have a critical role in immunity, and using TNF therapy has been reported to improve GI microbiota profile in IBD patients, treating IBD patients with COVID-19 using low-risk immune-based approaches could be a sensible way forward.

Acknowledgments. We thank the Inflammatory Bowel Disease Research Group and Microbiome Research group at King Abdulaziz University for discussion and constructive criticisms on drafts of this article.

Conflict of interests. The authors declare no conflict of interests.

Funding. No funding was received.

\section{REFERENCES}

[1] Al-Ani, A., Prentice, R., Rentsch, C., Johnson, D., Ardalan, Z., Heerasing, N., Garg, M., Campbell, S., Sasadeusz, J., Macrae, F. (2020): Prevention, diagnosis and management of COVID-19 in the inflammatory bowel disease patient. - Alimentary Pharmacology \& Therapeutics. https://doi.org/10.1111/apt.15779.

[2] Al-Muharraqi, M. A. (2020): Testing recommendation for COVID-19 (SARS-CoV-2) in patients planned for surgery - continuing the service and 'suppressing' the pandemic. $-\mathrm{Br}$ J Oral Maxillofac Surg 58(5): 503-505.

[3] Alifano, M., Alifano, P., Forgez, P., Iannelli, A. (2020): Renin-angiotensin system at the heart of COVID-19 pandemic. - Biochimie 174: 30-33.

[4] Alshehri, D., Saadah, O., Mosli, M., Edris, S., Alhindi, R., Bahieldin, A. (2020): Dysbiosis of gut microbiota in inflammatory bowel disease: current therapies and potential for microbiota-modulating therapeutic approaches. - Bosnian Journal of Basic Medical Sciences. https://doi.org/10.17305/bjbms.2020.5016.

[5] Arboleda, J. F., Fernandez, G. J., Urcuqui-Inchima, S. (2019): Vitamin D-mediated attenuation of miR-155 in human macrophages infected with dengue virus: implications for the cytokine response. - Infection, Genetics and Evolution 69: 12-21.

[6] Beaugerie, L., Rahier, J.-F., Kirchgesner, J. (2020): Predicting, preventing, and managing treatment-related complications in patients with inflammatory bowel diseases. - Clinical Gastroenterology and Hepatology. doi: 10.1016/j.cgh.2020.02.009.

[7] Bingula, R., Filaire, M., Radosevic-Robin, N., Bey, M., Berthon, J.-Y., BernalierDonadille, A., Vasson, M.-P., Filaire, E. (2017): Desired turbulence? Gut-lung axis, immunity, and lung cancer. - Journal of Oncology. https://doi.org/10.1155/2017/5035371.

[8] Cao, W., Li, T. (2020): COVID-19: towards understanding of pathogenesis. - Cell Research 30(5): 367-369.

[9] Cascella, M., Rajnik, M., Cuomo, A., Dulebohn, S. C., Di Napoli, R. (2020): Features, Evaluation and Treatment Coronavirus (COVID-19). - StatPearls Publishing, Tampa, FL.

[10] Celiberto, L. S., Graef, F. A., Healey, G. R., Bosman, E. S., Jacobson, K., Sly, L. M., Vallance, B. A. (2018): Inflammatory bowel disease and immunonutrition: novel therapeutic approaches through modulation of diet and the gut microbiome. Immunology 155(1): 36-52.

[11] Channappanavar, R., Fehr, A. R., Vijay, R., Mack, M., Zhao, J., Meyerholz, D. K., Perlman, S. (2016): Dysregulated type I interferon and inflammatory monocyte- 
macrophage responses cause lethal pneumonia in SARS-CoV-infected mice. - Cell Host \& Microbe 19(2): 181-193.

[12] Cheung, K. S., Hung, I. F., Chan, P. P., Lung, K., Tso, E., Liu, R., Ng, Y., Chu, M. Y., Chung, T. W., Tam, A. R. (2020): Gastrointestinal manifestations of SARS-CoV-2 infection and virus load in fecal samples from the Hong Kong cohort and systematic review and meta-analysis. https://doi.org/10.1053/j.gastro.2020.03.065

[13] Claesson, M. J., Cusack, S., O’Sullivan, O., Greene-Diniz, R., de Weerd, H., Flannery, E., Marchesi, J. R., Falush, D., Dinan, T., Fitzgerald, G. (2011): Composition, variability, and temporal stability of the intestinal microbiota of the elderly. - Proceedings of the National Academy of Sciences 108(Supplement 1): 4586-4591.

[14] Claesson, M. J., Jeffery, I. B., Conde, S., Power, S. E., O'connor, E. M., Cusack, S., Harris, H. M., Coakley, M., Lakshminarayanan, B., O'Sullivan, O. (2012): Gut microbiota composition correlates with diet and health in the elderly. - Nature 488(7410): 178-184.

[15] Conti, P., Ronconi, G., Caraffa, A., Gallenga, C., Ross, R., Frydas, I., Kritas, S. (2020): Induction of pro-inflammatory cytokines (IL-1 and IL-6) and lung inflammation by Coronavirus-19 (COVI-19 or SARS-CoV-2): anti-inflammatory strategies. - J Biol Regul Homeost Agents 34(2): 1.

[16] Danese, S., Cecconi, M., Spinelli, A. (2020): Management of IBD during the COVID-19 outbreak: resetting clinical priorities. - Nature Reviews Gastroenterology \& Hepatology 17(5): 253-255.

[17] De Musis, C., Granata, L., Dallio, M., Miranda, A., Gravina, A. G., Romano, M. (2020): Inflammatory bowel diseases: the role of gut microbiota. - Current Pharmaceutical Design. DOI: 10.2174/1381612826666200420144128.

[18] Dipasquale, V., Romano, C. (2018): Pharmacological treatments and infectious diseases in pediatric inflammatory bowel disease. - Expert Review of Gastroenterology \& Hepatology 12(3): 237-247.

[19] Dumas, A., Bernard, L., Poquet, Y., Lugo-Villarino, G., Neyrolles, O. (2018): The role of the lung microbiota and the gut-lung axis in respiratory infectious diseases. - Cellular Microbiology 20(12): e12966.

[20] Effenberger, M., Grabherr, F., Mayr, L., Schwaerzler, J., Nairz, M., Seifert, M., Hilbe, R., Seiwald, S., Scholl-Buergi, S., Fritsche, G. (2020): Faecal calprotectin indicates intestinal inflammation in COVID-19. - Gut. http://dx.doi.org/10.1136/gutjnl-2020-321388.

[21] Feldmann, M., Maini, R. N., Woody, J. N., Holgate, S. T., Winter, G., Rowland, M., Richards, D., Hussell, T. (2020): Trials of anti-tumour necrosis factor therapy for COVID-19 are urgently needed. - The Lancet 395(10234): 1407-1409.

[22] Ford, A. C., Peyrin-Biroulet, L. (2013): Opportunistic infections with anti-tumor necrosis factor- $\alpha$ therapy in inflammatory bowel disease: meta-analysis of randomized controlled trials. - American Journal of Gastroenterology 108(8): 1268-1276.

[23] Gao, J., Xu, K., Liu, H., Liu, G., Bai, M., Peng, C., Li, T., Yin, Y. (2018): Impact of the gut microbiota on intestinal immunity mediated by tryptophan metabolism. - Frontiers in Cellular and Infection Microbiology 8: 13.

[24] Garg, M., Royce, S. G., Tikellis, C., Shallue, C., Batu, D., Velkoska, E., Burrell, L. M., Patel, S. K., Beswick, L., Jackson, A. (2020): Imbalance of the renin-angiotensin system may contribute to inflammation and fibrosis in IBD: a novel therapeutic target? - Gut 69(5): 841-851.

[25] Ghosh, S., Klein, R. S. (2017): Sex drives dimorphic immune responses to viral infections. - The Journal of Immunology 198(5): 1782-1790.

[26] Gilbert, J. A., Blaser, M. J., Caporaso, J. G., Jansson, J. K., Lynch, S. V., Knight, R. (2018): Current understanding of the human microbiome. - Nature Medicine 24: 392.

[27] Gong, J., Dong, H., Xia, S. Q., Huang, Y. Z., Wang, D., Zhao, Y., Liu, W., Tu, S., Zhang, M., Wang, Q. (2020): Correlation analysis between disease severity and inflammation- 
related parameters in patients with COVID-19 pneumonia. - MedRxiv. https://doi.org/10.1101/2020.02.25.20025643.

[28] Grant, W. B., Lahore, H., McDonnell, S. L., Baggerly, C. A., French, C. B., Aliano, J. L., Bhattoa, H. P. (2020): Evidence that vitamin D supplementation could reduce risk of influenza and COVID-19 infections and deaths. - Nutrients 12(4): 988.

[29] Greiller, C. L., Martineau, A. R. (2015): Modulation of the immune response to respiratory viruses by vitamin D Nutrients 7(6): 4240-4270.

[30] Groves, H. T., Higham, S. L., Moffatt, M. F., Cox, M. J., Tregoning, J. S. (2020): Respiratory viral infection alters the gut microbiota by inducing inappetence. - Mbio 11(1).

[31] Guo, Y.-R., Cao, Q.-D., Hong, Z.-S., Tan, Y.-Y., Chen, S.-D., Jin, H.-J., Tan, K.-S., Wang, D.-Y., Yan, Y. (2020): The origin, transmission and clinical therapies on coronavirus disease 2019 (COVID-19) outbreak-an update on the status. - Military Medical Research 7(1): 1-10.

[32] Hoffmann, M., Kleine-Weber, H., Schroeder, S., Krüger, N., Herrler, T., Erichsen, S., Schiergens, T. S., Herrler, G., Wu, N.-H., Nitsche, A., Müller, M. A., Drosten, C., Pöhlmann, S. (2020): SARS-CoV-2 Cell entry depends on ACE2 and TMPRSS2 and is blocked by a clinically proven protease inhibitor. - Cell 181(2): 271-280.e278.

[33] Holmer, A., Singh, S. (2019): Overall and comparative safety of biologic and immunosuppressive therapy in inflammatory bowel diseases. - Expert review of clinical immunology 15(9): 969-979.

[34] Holshue, M. L., DeBolt, C., Lindquist, S., Lofy, K. H., Wiesman, J., Bruce, H., Spitters, C., Ericson, K., Wilkerson, S., Tural, A. (2020): First case of 2019 novel coronavirus in the United States. - New England Journal of Medicine. DOI: 10.1056/NEJMoa2001191.

[35] Huang, C., Wang, Y., Li, X., Ren, L., Zhao, J., Hu, Y., Zhang, L., Fan, G., Xu, J., Gu, X. (2020): Clinical features of patients infected with 2019 novel coronavirus in Wuhan, China. - The Lancet 395(10223): 497-506.

[36] Ibrahim, I. M., Abdelmalek, D. H., Elshahat, M. E., Elfiky, A. A. (2020): COVID-19 spike-host cell receptor GRP78 binding site prediction. - Journal of Infection. https://doi.org/10.1016/j.jinf.2020.02.026.

[37] Jablaoui, A., Kriaa, A., Mkaouar, H., Akermi, N., Soussou, S., Wysocka, M., Wołoszyn, D., Amouri, A., Gargouri, A., Maguin, E. (2020): Fecal serine protease profiling in inflammatory bowel diseases. - Frontiers in Cellular and Infection Microbiology 10: 21.

[38] Keely, S., Talley, N. J., Hansbro, P. M. (2012): Pulmonary-intestinal cross-talk in mucosal inflammatory disease. - Mucosal Immunology 5(1): 7-18.

[39] Khan, I., Ullah, N., Zha, L., Bai, Y., Khan, A., Zhao, T., Che, T., Zhang, C. (2019): Alteration of gut microbiota in inflammatory bowel disease (IBD): cause or consequence? IBD treatment targeting the gut microbiome. - Pathogens 8(3): 126.

[40] Kong, J., Zhu, X., Shi, Y., Liu, T., Chen, Y., Bhan, I., Zhao, Q., Thadhani, R., Li, Y. C. (2013): VDR attenuates acute lung injury by blocking Ang-2-Tie-2 pathway and reninangiotensin system. - Molecular Endocrinology 27(12): 2116-2125.

[41] Kovalchuk, A., Rodriguez-Juarez, R., Ilnytskyy, S., Li, D., Wang, B., Kovalchuk, I., Kovalchuk, O. (2020): Fighting the storm: novel anti-TNF $\alpha$ and anti-IL-6 C sativa lines to tame cytokine storm in COVID-19. - Europe PMC. DOI: 10.21203/rs.3.rs-30927/v1.

[42] Kweder, H., Eidi, H. (2018): Vitamin D deficiency in elderly: risk factors and drugs impact on vitamin D status. - Avicenna J Med 8(4): 139-146.

[43] Lagkouvardos, I., Overmann, J., Clavel, T. (2017): Cultured microbes represent a substantial fraction of the human and mouse gut microbiota. - Gut Microbes 8(5): 493503.

[44] Lai, M. M., Cavanagh, D. (1997): The molecular biology of coronaviruses. - Advances in Virus Research. https://doi.org/10.1016/S0065-3527(06)66005-3.

[45] Lake, M. A. (2020): What we know so far: COVID-19 current clinical knowledge and research. - Clinical Medicine 20(2): 124. 
[46] Lavelle, A., Sokol, H. (2020): Gut microbiota-derived metabolites as key actors in inflammatory bowel disease. - Nature Reviews Gastroenterology \& Hepatology 17: 223237.

[47] Leung, W. K., To K-f, Chan, P. K., Chan, H. L., Wu, A. K., Lee, N., Yuen, K. Y., Sung, J. J. (2003): Enteric involvement of severe acute respiratory syndrome-associated coronavirus infection. - Gastroenterology 125(4): 1011-1017.

[48] Li, D., Yu, G., Hu, H., Li, X., Wang, Y., Xiao, Y., Zhang, T., Gao, X., Ghozlane, A. (2017): Characteristics of faecal microbiota in paediatric Crohn's disease and their dynamic changes during infliximab therapy. - Journal of Crohn's and Colitis 12(3): 337346.

[49] Lichtenstein, G. R., Feagan, B. G., Cohen, R. D., Salzberg, B. A., Diamond, R. H., Price, S., Langholff, W., Londhe, A., Sandborn, W. J. (2012): Serious infection and mortality in patients with Crohn's disease: more than 5 years of follow-up in the TREAT ${ }^{\mathrm{TM}}$ registry. The American Journal of Gastroenterology 107(9): 1409.

[50] Lin, L., Jiang, X., Zhang, Z., Huang, S., Zhang, Z., Fang, Z., Gu, Z., Gao, L., Shi, H., Mai, L. (2020): Gastrointestinal symptoms of 95 cases with SARS-CoV-2 infection. Gut 69(6): 997-1001.

[51] Liu, K., Chen, Y., Lin, R., Han, K. (2020): Clinical features of COVID-19 in elderly patients: a comparison with young and middle-aged patients. - Journal of Infection 80(6): e14-e18.

[52] Long, M. D., Martin, C., Sandler, R. S., Kappelman, M. D. (2013): Increased risk of pneumonia among patients with inflammatory bowel disease. - The American Journal of Gastroenterology 108(2): 240.

[53] Lu, R., Zhao, X., Li, J., Niu, P., Yang, B., Wu, H., Wang, W., Song, H., Huang, B., Zhu, N. (2020): Genomic characterisation and epidemiology of 2019 novel coronavirus: implications for virus origins and receptor binding. - The Lancet 395(10224): 565-574.

[54] McDermott, J. E., Mitchell, H. D., Gralinski, L. E., Eisfeld, A. J., Josset, L., Bankhead, A., Neumann, G., Tilton, S. C., Schäfer, A., Li, C. (2016): The effect of inhibition of PP1 and TNF $\alpha$ signaling on pathogenesis of SARS coronavirus. - BMC Systems Biology 10(1): 93.

[55] Monteleone, G., Ardizzone, S. (2020): Are patients with inflammatory bowel disease at increased risk for Covid-19 infection? - Journal of Crohn's and Colitis. https://doi.org/10.1093/ecco-jcc/jjaa061.

[56] Ning, L., Shan, G., Sun, Z., Zhang, F., Xu, C., Lou, X., Li, S., Du, H., Chen, H., Xu, G. (2019): Quantitative proteomic analysis reveals the deregulation of nicotinamide adenine dinucleotide metabolism and CD38 in inflammatory bowel disease. - BioMed Research International. DOI: 10.1155/2019/3950628.

[57] Panarese, A., Pesce, F., Porcelli, P., Riezzo, G., Iacovazzi, P. A., Leone, C. M., De Carne, M., Rinaldi, C. M., Shahini, E. (2019): Chronic functional constipation is strongly linked to vitamin D deficiency. - World Journal of Gastroenterology 25(14): 1729.

[58] Redd, W. D., Zhou, J. C., Hathorn, K. E., McCarty, T. R., Bazarbashi, A. N., Thompson, C. C., Shen, L., Chan, W. W. (2020): Prevalence and characteristics of gastrointestinal symptoms in patients with SARS-CoV-2 infection in the United States: a multicenter cohort study. - Gastroenterology. DOI: 10.1053/j.gastro.2020.04.045.

[59] Rico-Mesa, J. S., White, A., Anderson, A. S. (2020): Outcomes in patients with COVID19 infection taking ACEI/ARB. - Curr Cardiol Rep 22(5): 31-31.

[60] Sartor, R. B. (2006): Mechanisms of disease: pathogenesis of Crohn's disease and ulcerative colitis. - Nature Clinical Practice Gastroenterology \& Hepatology 3(7): 390407.

[61] Schirmer, M., Smeekens, S. P., Vlamakis, H., Jaeger, M., Oosting, M., Franzosa, E. A., ter Horst, R., Jansen, T., Jacobs, L., Bonder, M. J., Kurilshikov, A., Fu, J., Joosten, L. A. B., Zhernakova, A., Huttenhower, C., Wijmenga, C., Netea, M. G., Xavier, R. J. (2016): 
Linking the human gut microbiome to inflammatory cytokine production capacity. - Cell 167(4): 1125-1136.e1128.

[62] Schwab, M., Reynders, V., Loitsch, S., Steinhilber, D., Stein, J., Schröder, O. (2007): Involvement of different nuclear hormone receptors in butyrate-mediated inhibition of inducible NFkB signalling. - Molecular Immunology 44(15): 3625-3632.

[63] Shah, E. D., Farida, J. P., Siegel, C. A., Chong, K., Melmed, G. Y. (2017): Risk for overall infection with anti-TNF and anti-integrin agents used in IBD: a systematic review and meta-analysis. - Inflammatory Bowel Diseases 23(4): 570-577.

[64] Singh, S., Facciorusso, A., Dulai, P. S., Jairath, V., Sandborn, W. J. (2020): Comparative risk of serious infections with biologic and/or immunosuppressive therapy in patients with inflammatory bowel diseases: a systematic review and meta-analysis. - Clinical Gastroenterology and Hepatology 18(1): 69-81. e63.

[65] Singhal, T. (2020): A review of coronavirus disease-2019 (COVID-19). - Indian J Pediatr 87(4): 281-286.

[66] Su, S., Wong, G., Shi, W., Liu, J., Lai, A. C., Zhou, J., Liu, W., Bi, Y., Gao, G. F. (2016): Epidemiology, genetic recombination, and pathogenesis of coronaviruses. - Trends in Microbiology 24(6): 490-502.

[67] Thorburn, A. N., Macia, L., Mackay, C. R. (2014): Diet, metabolites, and "westernlifestyle" inflammatory diseases. - Immunity 40(6): 833-842.

[68] Wang, D., Hu, B., Hu, C., Zhu, F., Liu, X., Zhang, J., Wang, B., Xiang, H., Cheng, Z., Xiong, Y. (2020): Clinical characteristics of 138 hospitalized patients with 2019 novel coronavirus-infected pneumonia in Wuhan, China. - JAMA 323(11): 1061-1069.

[69] Weiss, S. R., Navas-Martin, S. (2005): Coronavirus pathogenesis and the emerging pathogen severe acute respiratory syndrome coronavirus. - Microbiol. Mol. Biol. Rev. 69(4): 635-664.

[70] White, J. H. (2018): Vitamin D deficiency and the pathogenesis of Crohn's disease. - The Journal of Steroid Biochemistry and Molecular Biology 175: 23-28.

[71] Wölfel, R., Corman, V. M., Guggemos, W., Seilmaier, M., Zange, S., Müller, M. A., Niemeyer, D., Jones, T. C., Vollmar, P., Rothe, C. (2020): Virological assessment of hospitalized patients with COVID-2019. - Nature 581(7809): 465-469.

[72] Wu, D., Yang, X. O. (2020): TH17 responses in cytokine storm of COVID-19: An emerging target of JAK2 inhibitor Fedratinib. - Journal of Microbiology, Immunology and Infection 53(3): 368-370.

[73] Wu, Y., Guo, C., Tang, L., Hong, Z., Zhou, J., Dong, X., Yin, H., Xiao, Q., Tang, Y., Qu, X. (2020): Prolonged presence of SARS-CoV-2 viral RNA in faecal samples. - The Lancet Gastroenterology \& Hepatology 5(5): 434-435.

[74] Xiao, F., Tang, M., Zheng, X., Liu, Y., Li, X., Shan, H. (2020): Evidence for gastrointestinal infection of SARS-CoV-2. - Gastroenterology 158(6): 1831-1833. e1833.

[75] Yatsunenko, T., Rey, F. E., Manary, M. J., Trehan, I., Dominguez-Bello, M. G., Contreras, M., Magris, M., Hidalgo, G., Baldassano, R. N., Anokhin, A. P. (2012): Human gut microbiome viewed across age and geography. - Nature 486(7402): 222-227.

[76] Ye, Q., Wang, B., Mao, J. (2020): The pathogenesis and treatment of the 'Cytokine Storm' in COVID-19. - Journal of Infection 80(6): 607-613.

[77] Zang, R., Castro, M. F. G., McCune, B. T., Zeng, Q., Rothlauf, P. W., Sonnek, N. M., Liu, Z., Brulois, K. F., Wang, X., Greenberg, H. B. (2020): TMPRSS2 and TMPRSS4 promote SARS-CoV-2 infection of human small intestinal enterocytes. - Science Immunology 5(47).

[78] Zdrenghea, M. T., Makrinioti, H., Bagacean, C., Bush, A., Johnston, S. L., Stanciu, L. A. (2017): Vitamin D modulation of innate immune responses to respiratory viral infections. - Reviews in Medical Virology 27(1): e1909.

[79] Zhang, Y., Leung, D. Y., Richers, B. N., Liu, Y., Remigio, L. K., Riches, D. W., Goleva, E. (2012): Vitamin D inhibits monocyte/macrophage proinflammatory cytokine 
production by targeting MAPK phosphatase-1. - The Journal of Immunology 188(5): 2127-2135.

[80] Zhang, D., Li, S., Wang, N., Tan, H.-Y., Zhang, Z., Feng, Y. (2020): The cross-talk between gut microbiota and lungs in common lung diseases. - Frontiers in Microbiology 11. DOI: $10.3389 / \mathrm{fmicb} .2020 .00301$.

[81] Zhang, W., Du, R.-H., Li, B., Zheng, X.-S., Yang, X.-L., Hu, B., Wang, Y.-Y., Xiao, G.F., Yan, B., Shi, Z.-L. (2020): Molecular and serological investigation of 2019-nCoV infected patients: implication of multiple shedding routes. - Emerging Microbes \& Infections 9(1): 386-389.

[82] Zhou, P., Yang, X.-L., Wang, X.-G., Hu, B., Zhang, L., Zhang, W., Si, H.-R., Zhu, Y., Li, B., Huang, C.-L. (2020): A pneumonia outbreak associated with a new coronavirus of probable bat origin. - Nature 579(7798): 270-273.

[83] Zhu, N., Zhang, D., Wang, W., Li, X., Yang, B., Song, J., Zhao, X., Huang, B., Shi, W., Lu, R. (2020): A novel coronavirus from patients with pneumonia in China, 2019. - New England Journal of Medicine. DOI: 10.1056/NEJMoa2001017. 